\section{Viviendo con trasplante renal, sin protección social en salud: ¿Qué dicen los enfermos sobre las dificultades económicas que enfrentan y sus efectos?}

\author{
Kidney transplant patients without social \\ protection in health: what do patients say about \\ the economic hardships and impact?
}

\section{Viver com transplante renal sem proteção social em saúde: o que os pacientes dizem sobre as dificuldades econômicas e os seus efeitos?}

Francisco Javier Mercado-Martínez 1 Eduardo Hernández-Ibarra 2

Carlos D. Ascencio-Mera 1

Blanca A. Díaz-Medina 1

Cesar Padilla-Altamira 3

Ciara Kierans 3

\footnotetext{
${ }^{1}$ Universidad de

Guadalajara, Guadalajara, México.

2 Facultad de Enfermería, Universidad Autónoma de

San Luis Potosí, San Luis Potosí, México.

3 University of Liverpool,

Liverpool, U.K.

Correspondencia F. J. Mercado-Martínez Universidad de Guadalajara. Mar Egeo 1452-41, Country Club, Guadalajara, Jalisco 44610, México. fjaviermercado@yahoo. com.mx
}

\begin{abstract}
Kidney transplant is the optimal treatment for renal disease according to biomedical criteria, but the technology is highly expensive. The aim of this article was to examine the economic hardships experienced by kidney transplant patients and the impact on their lives, specifically when they lack social protection in health. The article reports on a qualitative study conducted in Mexico. Twenty-one kidney transplant patients participated. Semi-structured interviews were performed and submitted to content analysis. Patients experience extreme economic hardship due to the high cost of renal therapies, particularly medicines. Such economic problems adversely affect their condition, since many patients report difficulties in maintaining their immunosuppressant medication, attending medical appointments, and curtailing household expenditures, further aggravated by loss of earnings. In conclusion, kidney transplantation is associated with patients' impoverishment when they lack social protection in health. A protection system is urgently needed for this group.
\end{abstract}

Chronic Renal Insufficiency; Kidney Transplantation; Social Inequity

\section{Resumen}

El trasplante es el mejor tratamiento para la insuficiencia renal, de acuerdo con la biomedicina; sin embargo, es una tecnología cara. Este artículo examina las dificultades económicas y sus efectos en personas con trasplante renal, pero sin protección social en salud. Para el estudio se desarrolló una investigación cualitativa en México. Participaron 21 pacientes trasplantados; se aplicaron entrevis tas semiestructuradas, y se realizó un análisis de contenido. Los resultados muestran que las personas enfermas enfrentan dificultades económicas, debido a los costes de las terapias renales, particularmente, de las medicinas. Todo ello tiene efectos negativos: las personas con pocos recursos dejan el tratamiento con medicamentos, abandonan el protocolo, no asisten a las consultas médicas y disminuyen los gastos domésticos, incluyendo el de alimentación; además de suponer una merma en los ingresos familiares. En conclusión, el trasplante renal empobrece a las personas enfermas sin protección social en salud; es urgente la implementación de un sistema de protección social para esta población.

Insuficiencia Renal Crónica; Trasplante de Riñon; Inequidad Social 


\section{Introducción}

El trasplante renal es el tratamiento óptimo de la insuficiencia renal crónica, según el modelo biomédico. Entre otros argumentos, porque mejora la calidad de vida de los enfermos y los reintegra a las actividades productivas y a una vida normal 1 . También se le considera la terapia renal más eficiente en términos de costo-efectividad, al tener menores costos que la diálisis 2 , disminuir la morbi-mortalidad ${ }^{3}$ y ahorrar gastos a los servicios de salud y a la sociedad en su conjunto 4 .

Los programas de trasplante renal, sin embargo, enfrentan múltiples dificultades, sobre todo económicas. Por un lado, es una terapia costosa 5 , tanto para los países desarrollados 6 , como para aquellos en vías de desarrollo 7,8. Por otro, la disponibilidad de recursos económicos tiene efectos en la actividad trasplantadora y sus resultados; por ejemplo, las tasas de supervivencia de los órganos trasplantados son mayores en países en los cuales las personas enfermas tienen acceso universal a los servicios de salud, al asegurarles la cobertura de los medicamentos inmunosupresores ${ }^{9}$. Además, un $70 \%$ de los 254 programas de trasplante renal en Estados Unidos reportan que los enfermos tienen problemas para pagar los medicamentos y que $68 \%$ de los programas presentan muertes o rechazo de órganos, debido a los costos de la terapia inmunosupresora 10 . Tales carencias parecen explicar que $50 \%$ de las personas con trasplante renal no sigan el tratamiento 11 , lo que podría dar cuenta de un $20 \%$ de los rechazos agudos de órganos y un $16 \%$ de los órganos perdidos 12 .

Algunos trabajos han examinado los obstáculos que enfrentan las personas trasplantadas en países con acceso universal a los servicios de salud; ninguno de ellos reporta haber encontrado problemas de tipo económico ${ }^{13}$. En cambio, en países con sistemas de salud fragmentados, las personas trasplantadas sin seguridad social difícilmente llevan a cabo el tratamiento, debido a sus costos económicos 14 . Por ejemplo, se ha documentado que en la India algunos trasplantados suspenden los medicamentos, debido a sus altos costos y la falta de recursos para conseguirlos 8 .

A pesar de lo anterior, poco se ha investigado sobre las dificultades económicas que enfrentan quienes han recibido un trasplante renal y no tienen acceso a un sistema universal de salud, o un seguro médico 15,16. El asunto reviste importancia en América Latina porque más del $20 \%$ de la población no cuenta con un sistema de protección social en salud, ni con recursos para cubrir las terapias renales 17 . El tema también es relevante en el marco de las políticas sanitarias sobre las tecnologías médicas de alta complejidad, la inequidad en el acceso a los recursos médicos y los estudios sobre la experiencia del padecimiento y la desigualdad social 18 .

Este estudio tiene como objetivo examinar las dificultades económicas que enfrentan y los efectos que éstas tienen en la vida de los enfermos con trasplante renal, que no cuentan con acceso gratuito a la atención sanitaria. Dos preguntas específicas animan el estudio, ¿cuáles han sido las dificultades económicas que han enfrentado los enfermos una vez trasplantados?, y ¿cuáles han sido los efectos de tales dificultades en su vida diaria?

\section{Dos notas sobre el contexto}

Tanto el sistema de salud, como la organización del trasplante de órganos, son semejantes en todo México. El sistema de salud mexicano es fragmentado e inequitativo, en tanto la mitad de la población tiene seguridad social, menos de un $10 \%$ acceso a la medicina privada y el resto a un sistema asistencial a cargo del Seguro Popular 19. Éste es un seguro de salud público y voluntario, que tiene cerca de 52 millones de personas afiliadas; pero que excluye la cobertura de enfermedades como la insuficiencia renal crónica y las terapias renales sustitutivas -hemodiálisis, diálisis peritoneal y trasplante renal 20. En consecuencia, los propios enfermos y sus familias se ven obligados a cubrir los costos de cualquier tratamiento renal.

México también enfrenta desafíos en la atención de las personas con insuficiencia renal crónica que han sido trasplantadas. Por un lado, 25.791 trasplantes renales fueron realizados en el país entre el 2000 y el 2009 21. La tasa de trasplante renal es de las más altas de Latinoamérica (20.4 por millón de habitantes), pero la misma se ha logrado al priorizar los trasplantes de vivo relacionados; un $79 \%$ de los trasplantes renales realizados en 2011 correspondieron a ellos, mientras que sólo un $21 \%$ fueron los de cadáver 22 .

\section{Metodología}

Llevamos a cabo un trabajo cualitativo en tres estados de México (Guanajuato, Jalisco y San Luis Potosí), los cuales se caracterizan por tener una sólida tradición, estructura y organización relacionada con los trasplantes 21 .

Utilizando un muestreo propositivo ${ }^{23}$, seleccionamos 21 personas con trasplante renal, que no tenían acceso a un seguro público o privado, ni recursos para pagar la terapia renal. Doce eran hombres y nueve mujeres; sus edades oscilaban 
entre 23 y 65 años, 15 vivían en ciudades y 6 en áreas rurales. Entre junio del 2010 y agosto del 2012, aplicamos entrevistas semi-estructuradas en las unidades hospitalarias, así como en las sedes de las asociaciones que los apoyan. Lo anterior se debe a que el lugar de la entrevista fue propuesto por los mismos participantes. Siete de ellos también decidieron que un familiar los acompañase durante la entrevista. Tanto el primer autor (FJ.M.-M.), como un coautor, estuvieron a cargo de las mismas. Su duración fue de una hora en promedio. Empleamos una guía de entrevista. Las siguientes cuestiones sirven para ejemplificar el tipo de preguntas formuladas: ¿me podría contar como ha sido su vida desde que le diagnosticaron la enfermedad?; ¿cuáles han sido los principales problemas que ha tenido desde que la trasplantaron?; ¿podría explicarme en qué consisten las dificultades que menciona?

Las entrevistas fueron transcritas en un procesador de texto. Decidimos realizar un análisis de contenido temático 24; para lo cual nos apoyamos en el programa de cómputo Ethnograph versión 6.0 (Qualis Research Associates, Salt Lake City, Estados Unidos) que facilita la organización de las entrevistas. Las transcripciones fueron trasladadas al Ethnograph; éste programa permite enumerar cada línea del texto. El primer y segundo autor revisaron las transcripciones línea por línea y las codificaron para identificar los temas emergentes. Posteriormente, hicieron la comparación de los códigos y los fragmentos de textos seleccionados hasta lograr un consenso superior al 80\%. La información se analizó hasta identificar los temas generales y los subtemas. Luego se seleccionaron fragmentos de las entrevistas para ejemplificar con citas textuales.

En esta investigación se cumplieron los principios éticos contenidos en la Declaración de Helsinki. El criterio para usar los pseudónimos fue identificando los nombres más comunes en la región y asignándolos al azar. El proyecto fue evaluado y aprobado por cinco comités de ética: de la Universidad de Guadalajara, de la University of Liverpool y de tres hospitales públicos donde reciben atención los informantes.

\section{Resultados}

De acuerdo con los participantes de este estudio, la situación que han enfrentado desde que fueron trasplantados discrepa del panorama que les prometieron los profesionales de la salud. Más que disminuir los gastos, volver a la vida normal y a las actividades productivas, o mejorar su calidad de vida, los enfermos destacan la imposibilidad de seguir las terapias médicas debido fundamentalmente a motivos económicos. Reconocen haber sido informados de la necesidad de tomar medicamentos de por vida, pero nunca habérseles notificado en detalle los problemas que enfrentarían después del trasplante. Al igual que otros participantes, María, una mujer joven, destaca la falta de coincidencia entre lo que le aseguraron y los resultados.

"Todos [me] decían 'vas a tener una mejor calidad de vida... vas a poder estudiar, a llevar tu vida normal y todo eso'... Ya después del trasplante me comentaron... 'que el riñón tiene cierto tiempo de vida y que unos medicamentos pueden ocasionarte otras enfermedades' y cosas así... [En aquel momento] yo iba con la necesidad de recuperarme, iba con la emoción, pues... [y fue hasta después] cuando me enteré sobre esto [sus efectos y sus costos]...".

Tan pronto fueron diagnosticadas, las personas entrevistadas se percataron que ni el sistema de salud, ni las instituciones sanitarias, ni los programas o servicios hospitalarios contaban con recursos para cubrir los gastos previos, durante o posteriores al trasplante. La tónica fue semejante en los tres estados estudiados, los profesionales de la salud les aseguraban estar preocupados por los costos del tratamiento y hacer esfuerzos para ayudarlos; sin embargo, terminaron reconociendo que dicha tarea estaba más allá de sus posibilidades y argumentando que el problema radicaba en que el Seguro Popular no cubría las terapias renales.

Teniendo en mente la propaganda del Seguro Popular en los medios de comunicación, en la cual se le presentaba como la mejor opción sanitaria para las familias sin seguridad social, la constatación de que excluía el tratamiento de la insuficiencia renal terminó generando entre los participantes una mezcla de decepción y frustración. Coincidiendo con la opinión de varios más, Jorge expresaba su malestar sobre el particular cuando decía:

“El Seguro Popular no cubre los trasplantes. Les cubre con algunos estudios a los pacientes antes de trasplantarse y cuando no los tienen catalogados como pacientes de nefrología... Nosotros no estamos en el Seguro Popular porque no te cubre [la insuficiencia renal crónica]... No tiene caso, es una mentira del Gobierno".

\section{Los problemas económicos}

Según los participantes, múltiples obstáculos enfrentaron que les han impedido volver a la vida normal y a las actividades productivas. Entre otros mencionan el malestar físico, la calidad deficiente de la atención médica, la desorganización en los hospitales o los efectos colaterales 
de los medicamentos. Sin embargo, el problema económico ha sido el más apremiante. Para todos ellos, la enfermedad renal pasó a significar un tratamiento largo, complejo y costoso.

Las dificultades para cubrir los costos del tratamiento no se produjeron durante o después del trasplante. Según los participantes, comenzaron desde el momento en que les hicieron el diagnóstico y les indicaron una terapia renal. Así, tales dificultades se fueron presentando a lo largo proceso, primero durante la terapia dialítica, luego en el protocolo de evaluación, incrementándose el mes del trasplante y más adelante durante el mantenimiento. El protocolo de evaluación consiste en una serie de exámenes médicos que permiten determinar las condiciones específicas del paciente y la utilidad o no del trasplante 25 . Los gastos, en consecuencia, se fueron haciendo acumulativos. La madre de una joven con trasplante renal recuerda las dificultades económicas que enfrentaron desde la hemodiálisis.

"Es muy fea esta enfermedad. Ya estábamos asustados con ella, porque no teníamos facilidades de hacer hemodiálisis cada tercer día. Son 1.300 pesos por semana [12,50 pesos x 1 US dólar]. Luego ésta [hija], se me internaba mucho... El Seguro [Popular] no le ayuda a uno. Ahí [en el hospital público] derechito tiene uno que pagar".

Sin embargo, a partir del trasplante los gastos se incrementaron, a la par que las preocupaciones. Además de continuar con los de la hemodiálisis o la diálisis peritoneal, los enfermos se vieron obligados a pagar simultáneamente consultas, exámenes e interconsultas, en una carrera interminable de visitas a los médicos y los laboratorios para cumplir con el protocolo en el tiempo establecido; de otra forma tendrían que reiniciar el proceso. Ello sin dejar de lado otros gastos como los del hogar, la alimentación y el transporte. Lucía se refiere a las dificultades que enfrentó durante los meses que duró la etapa del protocolo.

"Económicamente ha sido muy duro... Yo siento la presión económica, porque tienes que terminar los estudios antes de tal fecha... Tienes que hacer tu alcancía para luego gastarlo todo y pensar también en los gastos del hogar, en la comida, el gas, la luz y cosas así. Los gastos se te amontonan en el periodo del trasplante".

$\mathrm{Y}$ si las dificultades económicas habían sido muchas hasta ese momento, es cuando se lleva a cabo el trasplante cuando aumentan exponencialmente. La cantidad varía ligeramente de un hospital a otro, en los distintos estados, según la condición de la familia y la capacidad de negociación del enfermo o su familiar; pero varios participantes sostienen haber pagado cerca de 90 mil pesos (7.500 USD) por el trasplante renal, a pesar de haberse llevado a cabo en un hospital público. Todo ello, a pesar de haberle notificado al personal de trabajo social que no contaban con los recursos, ni con ahorros, para cubrir tal cantidad.

No acababan de pagar el trasplante cuando los enfermos se enteraban de que había otros gastos adicionales. Si bien disminuyó el número de consultas y exámenes, y desaparecieron las sesiones de hemodiálisis, surgió la necesidad impostergable de conseguir los medicamentos inmunosupresores. El precio variaba según el lugar donde se consiguieran, pero su costo fue más elevado durante los primeros meses, por la falta de información sobre las opciones existentes. Varios participantes refieren haber pagado cerca de 6 mil pesos al mes sólo por tales medicamentos. Si los gastos de la terapia dialítica y del trasplante habían consumido parcial o totalmente los recursos de los enfermos y sus familias, los gastos a partir de este momento rebasaban su capacidad económica por lo cual pasaron a depender de las ayudas de terceros. Al igual que les ocurrió a otros participantes, Luis desconocía la gravedad del asunto percatándose hasta demasiado tarde.

“Después [del trasplante] viene lo más pesado económicamente... los medicamentos. Yo no sabía, sino hasta cuando le pregunté a una enfermera en la hemodiálisis 'Oye, cuando me trasplanten ¿ya nomas es el trasplante y igracias!, ;adiós!?.' 'No' dice 'tienes que estar tomando medicamento de por vida...' Sí, ya tiene que ser de por vida. Y la verdad es muy caro".

Un caso típico sobre el monto de los gastos de estas familias es el de un joven con insuficiencia renal crónica, a quien se le trasplantó un riñón. Según el relato de su madre, la familia hizo esfuerzos extraordinarios para cubrir los gastos del tratamiento a lo largo de un año. Acompañando el relato con las facturas, los gastos durante la hemodiálisis fueron de 11 mil pesos el primer mes, ya que las sesiones se hicieron en unidades privadas al no haber espacio en el hospital público. El segundo mes disminuyeron a 4 mil 300 pesos al haber encontrado espacio para la hemodiálisis en un hospital público. Durante los cinco meses del protocolo la cifra ascendió a 14 mil pesos mensuales, al combinarse la hemodiálisis con las consultas y los estudios correspondientes. En cambio, la cifra ascendió a 93 mil pesos el mes en que se llevó a cabo el trasplante. Y los gastos del primer mes pos-trasplante fueron de 19.400 pesos. En total, los gastos directos que se hicieron en este caso fueron superiores a 230 mil pesos (18.400 USD) durante ese año.

Los gastos hasta ahora referidos no contemplan otros gastos que las personas enfermas hicieron a lo largo del proceso. Entre ellos destacan la alimentación, el alojamiento y el transporte, 
tanto del enfermo, como del acompañante en las incontables visitas al hospital, a unidades de laboratorio y a especialistas, así como durante las hospitalizaciones. Estos gastos fueron mencionados con frecuencia y se convirtieron en objeto de preocupación, sobre todo por quienes no vivían en la ciudad donde se ubicaba el hospital. De hecho, la falta de recursos para cubrirlos se convirtieron en detonadores del incumplimiento de las terapias prescritas. A ello se refería Luis cuando señalaba.

“...la principal complicación de la falta de recursos es el rechazo [del órgano trasplantado]. Es la falta de inmunosupresores, la falta de capacidad para hacerse exámenes de laboratorio, la falta de transporte... [Incluso] mucha gente ya no tiene 300 pesos para venirse en autobús de fuera de la ciudad".

\section{Los efectos}

La situación económica de los participantes tiene efectos, tanto en el tratamiento médico, como en su vida diaria. De entrada, los enfermos que no cuentan con suficientes recursos enfrentan dificultades para trasladarse a la ciudad y, por ende, faltan a sus citas médicas y no compran medicamentos, por lo cual dejan de tomarlos. Aquellos con menos recursos son los primeros en abandonar el protocolo o el tratamiento. En algunos casos los obtienen en el mercado negro de medicamentos, pese a su dudosa calidad y procedencia. Juan se refiere a la falta de recursos y sus efectos en los términos siguientes.

“...yo tenía mi cita [con el médico] desde el otro año, pero no he podido venir por falta de dinero. Y ahora sí... la necesidad del medicamento [me obligó a venir]".

Varios participantes reconocen que en ocasiones cuentan con suficientes recursos para el pago de los estudios de laboratorio y las consultas, pero no disponen de medios adicionales para comer o el pago del transporte. En estas circunstancias deciden priorizar el pago de los servicios médicos, tal como lo refiere la madre de una enferma.

"Si nos piden estudios a veces dejo de comprar [la despensa]. Cuando sé que me voy a venir para acá [al hospital], una semana antes yo no compro [despensa]... de hecho ni salgo de paseo. Lo que me voy a gastar al centro lo voy a guardar para sus estudios".

Quienes han sido trasplantados también manifiestan permanentemente su temor ante el posible rechazo del órgano por la falta de medicamentos. Tal temor obedece a las dificultades para conseguir específicamente los inmunosupresores. Miguel expone su temor, semejante al que pasan muchos otros, al no contar con los recursos necesarios para la compra de los medicamentos.

"Así he andado toda la mañana [buscando los medicamentos]. He andado así y hasta ahorita no he conseguido nada... Y ya me queda poca medicina. Si uno la deja de tomar, otra vez vuelve uno a lo mismo [a la diálisis]”.

Quienes no cuentan $\mathrm{u}$ obtienen suficientes recursos económicos son los primeros en ser excluidos de las terapias renales ante la imposibilidad de continuar cubriendo los costos. Los comentarios de un joven enfermo y de una madre, cuya hija fue trasplantada, ejemplifican dos mecanismos que evidencian como los trasplantes están prácticamente vedados en los hospitales públicos a la población con mayores carencias económicas.

“Si tienes dinero todo se hace rápido... A uno le dan a entender [en el hospital] que el que tiene dinero avanza y el que no, no".

"Quitaron a una niña [del protocolo] porque su mamá no le echó ganas [consiguiendo recursos]. La quitaron y a mí me dolió que la quitaran. Iba a entrar la mía, pero me dolió que quitaran a la otra niña. La niña falleció y todo porque su mamá no le echó ganas. Yo escuché [al médico] cuando dijo 'quiten a (...) no le echa ganas, metan a (...) [al protocolo de trasplantes]"'.

Las dificultades económicas que enfrentan los enfermos no sólo se deben a los costos de la terapia renal. Las mismas también se dan por la disminución de los ingresos, tanto de quien padece, como de quienes le rodean. Varios participantes refieren haber dejado de trabajar durante la diálisis peritoneal, la hemodiálisis, el protocolo y después del trasplante, debido a los viajes frecuentes y las estancias en los hospitales o el malestar generalizado a causa de la enfermedad o el tratamiento. Aunado a ello, los familiares encargados del cuidado también dejaron de trabajar, total o parcialmente, porque los acompañaban a consulta, a los hospitales, a los laboratorios, y sobre todo, en la búsqueda de apoyos. La madre de una joven enferma, que vivía al interior del estado, aludía a tales dificultades cuando acompañaba a su hija trasplantada.

"Normalmente venimos [a la ciudad] de entrada por salida, pero si no hay manera de irnos nos tenemos que quedar... Llegamos el lunes [pasado], y llevamos ya más de ocho días... Teníamos que volver a estar aquí... nos volvieron a citar para el laboratorio el viernes y hoy a consulta. Le digo 'No mi' ja, pues nos quedamos porque ‘ćómo le hacemos para regresarnos?' Es bien difícil, bien pesado. Allá tengo mis hijos en la casa, solos, porque ni su papá está...”.

La promesa de reincorporarse a las actividades productivas y tener un ingreso estable, por 
otra parte, dista de hacerse realidad, según varios participantes. Un motivo consistía en el rechazo social a contratar a las personas trasplantadas, otro es la necesidad de dedicar tiempo y energía a conseguir medicamentos gratuitos o de bajo costo en organismos públicos o en asociaciones civiles, a asistir a consultas médicas y a cumplir las indicaciones de los profesionales de la salud o las asociaciones. La descripción de Luis ilustra los desafíos que enfrentan ante el rechazo a contratarlos.

"Cuando van [los enfermos a] buscar trabajo... el empleador [piensa] 'esta persona va a faltar mucho o cada rato va a estar hospitalizada. Cuando fui a buscar trabajo... las primeras veces... me preguntaban 'ite han operado de algo?'. Yo decía 'sí, me trasplantaron'. Veía su reacción en la cara, cambiaban la conversación y me decían 'bueno... luego te hablamos'. Me quedó como enseñanza no decirlo... De todos modos, no gano nada con ser sincero... Existe una barrera por parte del empleador [a contratar personas trasplantadas]".

\section{Discusión}

El propósito de este trabajo ha sido examinar las dificultades económicas que enfrentan los enfermos con trasplante renal, así como los efectos que las mismas tienen en sus vidas. El estudio se ha centrado particularmente en aquellos sin acceso gratuito a la atención sanitaria. Producto de una investigación con un diseño cualitativo, llevada a cabo en tres estados de México, el mismo no se propuso mostrar lo que ocurre en tales estados, ni en el país en su conjunto, por lo cual en ningún momento aspiró a generalizar los resultados, ni las conclusiones a esos ámbitos.

Aquí se ha intentado profundizar en el tema considerando el punto de vista de quienes viven con el padecimiento y suelen ser llamados "los beneficiarios de los programas de salud”. Si bien numerosos estudios se han realizado sobre los costos que representan los trasplantes para las instituciones de salud y la sociedad en su conjunto 26 , poco se ha explorado el tema desde la óptica de quienes han sido trasplantados y no cuentan con un sistema de protección social en salud. En este sentido, el presente trabajo es pionero en el tema no sólo para el caso mexicano, sino también para otros países de ingresos medios y bajos, en donde amplios sectores de la población no cuentan con seguridad social, ni con acceso gratuito y universal a un sistema de salud y, por ende, a las terapias renales 27.

Los hallazgos expuestos presentan un panorama complejo y desalentador que contrasta con la visión que suelen ofrecer los profesionales de la salud. Tal como lo ha documentado Crowley-Matoka 28 , estos profesionales trasmiten una imagen idílica en la cual quienes han sido trasplantados vuelven a la normalidad y a la vida productiva. En contraste con tal imagen, el hallazgo central de este trabajo muestra las dificultades económicas que enfrentan los enfermos trasplantados, debido a los costos de las terapias renales, aunado a la disminución de los ingresos. Para los enfermos que participaron en este estudio, que no cuentan con seguridad social, ni con recursos para pagar el tratamiento, el pago de una cifra cercana a 250 mil pesos (más de 20 mil USD) al año por los costos del proceso de un trasplante renal, en un hospital público, representa una cantidad fuera de sus posibilidades. Ello si se tiene en consideración que los ingresos de aquellos con un salario mínimo en México son menores a 2 mil pesos al mes 29. Esto significa que una persona con tal salario requeriría más de 10 años para pagar los gastos de un año de este tratamiento renal realizado en los servicios públicos de salud.

¿Por qué se reporta tan poco esta situación? Pareciera haber varias razones. Por una parte, la misma no se presenta en países con un sistema gratuito y universal de salud, el cual incluye la atención de las terapias renales. Por mencionar un caso, un estudio realizado en Reino Unido encuentra que si bien los enfermos trasplantados siguen motivados a seguir el tratamiento renal, también se sienten agotados, debido a lo largo del mismo ${ }^{30}$. Pero ninguna alusión se hace a los costos, ni la carga económica del trasplante, ya que disponen de ayuda social y total para el pago del tratamiento. Situaciones similares se reportan en otros países con un sistema único de salud que ofrece acceso gratuito y universal a las terapias renales, como en España 31 , Canadá 32 y Brasil 33,34.

Los resultados de nuestro estudio coinciden con los de quienes han indagado las dificultades económicas que enfrenta la población trasplantada sin acceso a los servicios de salud o a la seguridad social. Entre otros, trabajos realizados en Estados Unidos con personas sin seguridad reportan que enfrentan problemas financieros antes y después del trasplante renal y sobre todo dificultades para obtener los medicamentos 35 . Otros encuentran que la principal barrera para acceder al trasplante renal entre quienes no tienen seguridad social en Indonesia son los altos costos de los medicamentos 36 . En el mismo sentido, los costos elevados de los trasplantes en países de ingresos medios y bajos como India, Pakistán e Irán, representan un serio desgaste para los enfermos y sus familias, por lo cual tienen dificultades para seguir el tratamiento 7 . 
Recientemente, se ha calificado al Seguro Popular mexicano como la decisión correcta en términos éticos y la política más inteligente para proteger financieramente a la población 37 . Haciendo alusión al problema que podrían enfrentar más de 50 millones de mexicanos, sin seguridad social y, por ende, con acceso al mismo seguro, ni nos parece ético, ni una decisión inteligente la forma como excluye a miles de personas con insuficiencia renal crónica de los beneficios médico sociales. El problema de fondo pareciera radicar en que sistemas de protección a la salud, como el mexicano, se basan en principios ajenos a la equidad y solidaridad social, priorizando lo económico y la lógica del mercado más que el derecho a la salud 38,39. Así, en el caso que nos ocupa, seguir negando la atención médica a las personas con insuficiencia renal crónica significa negarle el derecho a la salud a un sector creciente de la población.

Varios autores han reportado que los costos del trasplante renal son menores que los de las terapias dialíticas y que representan ahorros para los sistemas de salud y a la sociedad en su conjunto ${ }^{40}$. Las evidencias presentadas en este trabajo muestran que sucede lo contrario, según las personas enfermas. Desde la óptica de quienes han sido trasplantados sin contar con acceso a la seguridad social, el asunto central radica en que el precio de los medicamentos inmunosupresores es superior a los costos de la hemodiálisis o la diálisis peritoneal. De hecho, el asunto se vuelve más complejo porque a partir del trasplante pasan a vivir con el temor permanente al rechazo del órgano, si no toman los medicamentos día a día, a diferencia de cuando estaban en alguna terapia dialítica. Para los participantes de este estudio, en consecuencia, vivir con el trasplante renal ha significado pasar a vivir permanentemente en medio de dificultades económicas.

Algunas líneas de investigación surgen de lo expuesto. Una remite a las razones por las cuales la comunidad académica manifiesta tan poco interés por el tema e, incluso, termina alabando las bondades del trasplante. ¿ $\mathrm{O}$ será cierta la aseveración de Hamdy ${ }^{41}$ (p. 6) cuando dice " pareciera que los expertos no se interesan en buscar la perspectiva de aquellos que más ganan o pierden con los trasplantes"? Otro tema son los efectos de la carencia de recursos económicos en la adherencia al tratamiento y en el rechazo del órgano trasplantado. En este sentido, en el futuro se podrían comparar las tasas de rechazo y la supervivencia de las personas que padecen enfer- medad renal, con y sin seguridad social, así como las de quienes cuentan con recursos propios y se trasplantan en hospitales privados. Una tercera línea sería indagar las estrategias y acciones que vienen empleando aquellos miles, que viviendo en la pobreza y sin seguridad social, terminan obteniendo los recursos para pagar el costo del trasplante y de los medicamentos inmunosupresores. En el mismo tenor estarían las acciones que vienen realizando las organizaciones sociales, particularmente las asociaciones civiles en la supervivencia de las personas trasplantadas.

Varias propuestas derivan de los resultados expuestos. A corto plazo urge informar claramente a enfermos y familiares sobre los costos y los efectos de las terapias renales, en este caso del trasplante, a fin de que dispongan de los elementos para tomar las decisiones correspondientes. Por otro lado, urge impulsar un debate público sobre quien debería pagar las terapias renales sustitutivas, en particular los trasplantes y los medicamentos inmunosupresores, de este sector de la población. También se requiere implementar un sistema universal, integral, equitativo, solidario y gratuito que garantice el derecho a la salud, tanto de la población con insuficiencia renal crónica, como con otras condiciones crónicas.

Tal como lo recuerdan Kasiske et al. 6, en la mitología griega los dioses castigaron a Tantalus provocándole una sed insaciable y dándole agua que se alejaba cada vez que trataba de tomarla. Hoy, pareciera que el castigo es semejante en el caso de las personas trasplantadas sin seguridad social, al ofrecerles "el regalo de vida" -un trasplante-, pero haciéndoles inalcanzables los costosos medicamentos inmunosupresores y el resto de la atención médica. Los casos aquí presentados, son un recordatorio no sólo del castigo que políticos, tomadores de decisiones y la sociedad en su conjunto siguen dando a quienes han sido trasplantados; sino también que se trata de un castigo ejemplar a quienes, padeciendo insuficiencia renal crónica, viven en la pobreza.

Para concluir, el estado mexicano sigue negándole el derecho a la salud a este sector de la población con enfermedad renal crónica. El trasplante renal está empobreciendo cada vez más a quienes -padeciendo esta condición- no cuentan con seguridad social. Urge, en consecuencia, generar mecanismos para garantizar el acceso gratuito de toda la población a las terapias renales. Se trata de un estudio realizado en México, pero que aplica en muchos otros países. 


\section{Resumo}

O transplante é o melhor tratamento para a insuficiência renal de acordo com a biomedicina; mas é uma tecnologia cara. Este artigo examina as dificuldades econômicas e os seus efeitos em pessoas com transplante renal, mas sem proteção social em saúde. Uma pesquisa qualitativa foi desenvolvida no México. Participaram 21 doentes transplantados; aplicaram-se entrevistas semiestruturadas, e foi realizada análise de conteúdo. Os resultados mostram que as pessoas doentes enfrentam dificuldades econômicas devido aos custos das terapias renais, particularmente das medicinas. Tudo isso tem efeitos negativos: as pessoas com poucos recursos abandonam o tratamento medicamentoso, deixam o protocolo, não comparecem às consultas médicas e diminuem as despensas domésticas, incluindo a comida; além da perda de ingressos. Em conclusão, o transplante renal empobrece as pessoas doentes sem proteção social em saúde; é urgente a implementação de um sistema de proteção social a esta população

Insuficiência Renal Crônica; Transplante de Rim; Iniquidade Social

\section{Colaboradores}

F. J. Mercado-Martínez participó en la concepción del estudio, trabajo de campo, análisis de datos, elaboración del manuscrito, revisión de edición. E. HernándezIbarra colaboró en la revisión de la literatura, trabajo de campo, análisis de los datos, redacción y revisión del artículo. C. D. Ascencio-Mera y B. A. Díaz-Medina participaron en la búsqueda y recuperación de la literatura, del trabajo de campo, procesamiento y análisis de datos y revisión del manuscrito. C. Padilla-Altamira colaboró en la búsqueda, recuperación y revisión de la literatura, trabajo de campo, análisis de datos y redacción del documento. C. Kierans participó de la concepción del estudio, análisis de los datos, redacción del documento y revisión final.

\section{Agradecimientos}

A las personas enfermas y sus familias, así como al personal de salud por su disposición y ayuda valiosa. Jorge Urías y Mauricio Correa apoyaron en la organización y manejo de la información. Verónica Mercado hizo la traducción al inglés y Juliana Zilmer al portugués. Dos evaluadores anónimos hicieron comentarios valiosos. El Consejo Nacional de Ciencia y Tecnología (CONACYT) de México brindó apoyo financiero para realizar el trabajo (000128950).

\section{Referencias}

1. Ogutmen B, Yildirim A, Sever M, Bozfakioglu S, Ataman R, Erek E, et al. Health-related quality of life after kidney transplantation in comparison intermittent hemodialysis, peritoneal dialysis, and normal controls. Transplant Proc 2006; 38:419-21.

2. Haller M, Gutjahr G, Kramar R, Harnoncourt F, Oberbauer R. Cost-effectiveness analysis of renal replacement therapy in Austria. Nephrol Dial Transplant 2011; 26:2988-95.

3. Oniscu G, Brown H, Forsythe J. Impact of cadaveric renal transplantation on survival in patients listed for transplantation. J Am Soc Nephrol 2005; 16:1859-65.

4. Howard K, Salkeld G, White S, McDonald S, Chadban S, Craig JC, et al. The cost-effectiveness of increasing kidney transplantation and home-based dialysis. Nephrology (Carlton) 2009; 14:123-32.

5. Gordon EJ, Prohaska TR, Sehgal AR. The financial impact of immunosuppressant expenses on new kidney transplant recipients. Clin Transplant 2008; 22:738-48
6. Kasiske BL, Cohen D, Lucey MR, Neylan JF. Payment for immunosuppression after organ transplantation. JAMA 2000; 283:2445-50.

7. Rizvi SA, Naqvi SA, Hussain Z, Hashmi A, Akhtar F, Hussain M, et al. Renal transplantation in developing countries. Kidney Int Suppl 2003; (83):S96-100.

8. Jha V. End-stage renal care in developing countries: the India experience. Ren Fail 2004; 26:201-8.

9. Gill JS, Tonelli M. Penny wise, pound foolish? Coverage limits on immunosuppression after kidney transplantation. N Engl J Med 2012; 366:586-9.

10. Evans RW, Applegate WH, Briscoe DM, Cohen DJ, Rorick CC, Murphy BT, et al. Cost-related immunosuppressive medication nonadherence among kidney transplant recipients. Clin J Am Soc Nephrol 2010; 5:2323-8.

11. Butler JA, Roderick P, Mullee M, Mason JC, Peveler RC. Frequency and impact of nonadherence to immunosuppressants after renal transplantation: a systematic review. Transplantation 2004; 77 : 769-76. 
12. Denhaerynck K, Dobbels F, Cleemput I, Desmyttere A, Schäfer-Keller P, Schaub S, et al. Prevalence, consequences, and determinants of nonadherence in adult renal transplant patients: a literature review. Transpl Int 2005; 18:1121-33.

13. Tong A, Howell M, Wong G, Webster AC, Howard K, Craig JC. The perspectives of kidney transplant recipients on medicine taking: a systematic review of qualitative studies. Nephrol Dial Transplant 2011; 26:344-54.

14. Pinsky B, Takemoto S, Lentine K, Burroughs T, Schnitzler M, Salvalaggio P. Transplant outcomes and economic costs associated with patient noncompliance to immunosuppression. Am J Transplant 2009; 9:2597-606.

15. Mizraji R, Alvarez I, Palacios RI, Fajardo C, Berrios C, Morales F, et al. Organ donation in Latin America. Transplant Proc 2007; 39:333-5.

16. Laederach-Hofmann K, Bunzel B. Noncompliance in organ transplant recipients: a literature review. Gen Hosp Psychiatry 2000; 22:412-24.

17. Rosenberg H, Andersson B. Repensar la protección social en salud. Rev Panam Salud Pública 2000; 8:118-25.

18. Nguyen VK, Peschard K. Anthropology, inequality, and disease: a review. Annu Rev Anthropol 2003; 32:447-74.

19. Laurell AC, Ronquillo JH. La segunda reforma de salud: aseguramiento y compra-venta de servicios. Salud Colect 2010; 6:137-48.

20. Comisión Nacional de Protección a la Salud. Seguro Popular de Salud. http://www.seguropopular. salud.gob.mx/index.php?option=com_content $\& v$ iew $=$ article\&id $=272 \& I t e m i d=287$ (accedido el 02/ $\mathrm{Feb} / 2013$ ).

21. Centro Nacional de Trasplantes. Estado actual de la donación y trasplante de órganos en México. Reporte Anual 2011. Delegación Tlalpan: Centro Nacional de Trasplantes; 2012.

22. Red/Consejo Iberoamericano de Donación y Trasplante. Actividad de donación y trasplante de órganos, tejidos y células y recomendaciones aprobadas por el Consejo Iberoamérica de Donación y Trasplante. Granada: Red/Consejo Iberoamericano de Donación y Trasplante; 2012.

23. Kuzel AJ. Sampling in qualitative inquiry. In: Crabtree BF, Miller WL, editors. Doing qualitative research. Newbury Park: Sage Publications; 1992. p. 31-44.

24. Hsieh H-F, Shannon SE. Three approaches to qualitative content analysis. Qual Health Res 2005; 15:1277-88.

25. Centro Nacional de Trasplantes. El proceso de trasplante. http://www.cenatra.salud.gob.mx/interior/ trasplante_proceso_trasplante.html (accedido el 19/Feb/2014).

26. Pacheco A, Saffie A, Torres R, Tortella C, Llanos C, Vargas D, et al. Cost/utility study of peritoneal dialysis and hemodialysis in Chile. Perit Dial Int 2007; 27:359-63.
27. Rofman R, Carranza E. La cobertura de la seguridad social en América Latina. http://cdi.mecon. gov.ar/biblio/docelec/az1089.pdf (accedido el 10/ Ene/2013).

28. Crowley-Matoka M. Desperately seeking "normal": the promise and perils of living with kidney transplantation. Soc Sci Med 2005; 61:821-31.

29. Comisión Nacional de Salarios Mínimos. Salario mínimo de los Estados Unidos Mexicano 19642014. http://www.conasami.gob.mx/pdf/salario minimo/sal_min_gral_prom.pdf (accedido el 24/ Ago/2012).

30. Orr A, Willis S, Holmes M, Britton P, Orr D. Living with a kidney transplant: a qualitative investigation of quality of life. J Health Psychol 2007; 12:653-62.

31. García PC, Montero MAC, Amblás RT. Cumplimiento del tratamiento en el trasplante renal pediátrico. Rev Soc Esp Enferm Nefrol 2010; 13:1139375.

32. Korus M, Stinson JN, Pool R, Williams A, Kagan S. Exploring the information needs of adolescents and their parents throughout the kidney transplant continuum. Progr Transplant 2011; 21:53-60.

33. Quintana AM, Weissheimer TKS, Hermann C. Atribuições de significados ao transplante renal. Psico (Porto Alegre) 2011; 42:23-30.

34. Arruda GO, Renovato RD. Uso de medicamentos em transplantados renais: práticas de medicação e representações. Rev Gaúch Enferm 2012; 33: 157-64.

35. Loghman-Adham M. Medication noncompliance in patients with chronic disease: issues in dialysis and renal transplantation. Am J Manag Care 2003; 9:155-71.

36. Bennett P, Hany A. Barriers to kidney transplants in Indonesia: a literature review. Int Nurs Rev 2009; 56:41-9.

37. Knaul FM, González-Pier E, Gómez-Dantés O, García-Junco D, Arreola-Ornelas H, Barraza-Lloréns $\mathrm{M}$, et al. The quest for universal health coverage: achieving social protection for all in Mexico. Lancet 2012; 380:1259-79.

38. Tamez S, Eibenschutz C. El Seguro Popular de Salud en México: pieza clave de la inequidad en salud. Rev Salud Pública 2008; 10 Suppl 1:133-45.

39. Laurell, AC. Health system reform in México: a critical review. Int J Health Serv 2007; 37:515-35.

40. Salonen T, Reina T, Oksa H, Sintonen H, Pasternack A. Cost analysis of renal replacement therapies in Finland. Am J Kidney Dis 2003; 42:1228-38.

41. Hamdy, S. Our bodies belong to God: organ transplants, Islam, and the struggle for human dignity in Egypt. Berkeley: University of California Press; 2012.

Recibido el 16/Sep/2013

Versión final presentada el 21/Feb/2014

Aprobado el 07/Mar/2014 